\title{
A DUAL TO THE BORDISM CONCEPT
}

\author{
R. E. STONG
}

Abstract. A dual to bordism is defined by reversing arrows. For finite complexes this Grothendieckizes to give $K O$ theory.

Let $X$ be a topological space. A drobism element of $X$ is a pair $(M, f)$ with $M$ a closed differentiable manifold and $f: X \rightarrow M$ a continuous map. Two drobism elements $(M, f)$ and $\left(M^{\prime}, f^{\prime}\right)$ are equivalent, or drobant, if there is a pair $(V, F)$ such that $V$ is a compact differentiable manifold with boundary, the boundary of $V$ being the disjoint union of $M$ and $M^{\prime}$, and $F: X \times I \rightarrow V$ is a continuous map with $F|X \times 0=f, F| X \times 1=f^{\prime}$. The set of equivalence classes of drobism elements of $X$ is called the drobism set of $X$ and will be denoted $(X) \mathfrak{N}_{*}$.

The set $(X) \mathfrak{N}_{*}$ is in a rather imprecise sense dual to the bordism of $X$, $\mathfrak{N}_{*}(X)$, as defined by Atiyah [1]. Specifically, if each map in the above is reversed one obtains the definition of bordism. This is vaguely the same as the relation between homotopy and cohomotopy, where again one simply reverses arrows, but is not a duality in a precise mathematical sense.

There is a product operation $\cdot(X) \mathfrak{N}_{*} \times(X) \mathfrak{N}_{*} \rightarrow(X) \mathfrak{N}_{*}$ defined by letting $[(M, f)] \cdot[(N, g)]$ be the class of the composite

$$
X \stackrel{\Delta}{\longrightarrow} X \times X \stackrel{f \times g}{\longrightarrow} M \times N
$$

where $\Delta$ is the diagonal map. This product is associative, commutative, and has a unit, given by the class of the map of $X$ to a point. There are not any inverses available (since in particular no negative dimensional manifolds exist).

One may then form a group associated with the semigroup $(X) \mathfrak{N}_{*}$, denoted by $\mathfrak{M}(X)$, by taking equivalence classes of pairs $(a, b), a, b \in(X) \mathfrak{N}_{*}$, with $(a, b) \equiv(c, d)$ if $a d=b c$. Then $(a, b) \cdot(c, d)=(a c, b d)$ gives $\mathfrak{M}(X)$ an operation making this set into an abelian group.

THEOREM. $\mathfrak{B}(X)$ is naturally isomorphic to $K O(X)$, if $X$ is a finite complex.

Received by the editors August 19, 1971.

AMS 1970 subject classifications. Primary 57D90; Secondary 55F50.

(c) American Mathematical Society 1972 
Proof. If $(M, f)$ is a drobism element of $X$, let $\phi(M, f) \in K O(X)$ be the class of the bundle $f^{*} \tau_{M}$, where $\tau_{M}$ is the tangent bundle of $M$. For a manifold with boundary $V, \tau_{V} \mid \partial V \cong \tau_{\partial V} \oplus 1$, so $\phi(M, f)$ depends only on the class of $(M, f)$. Since $\tau_{M \times N} \cong \tau_{M} \oplus \tau_{N}$, this defines a homomorphism

$$
\phi:(X) \mathfrak{N}_{*} \rightarrow K O(X)
$$

and by the associated group construction, a homomorphism

$$
\phi: \mathfrak{W}(X) \rightarrow K O(X) .
$$

If $X$ is a compact Hausdorff space, $\phi$ is epic, for let $\xi$ be a bundle over $X$, classified stably by a map $g: X \rightarrow G_{r, s}$ with $G_{r, s}$ the Grassmannian of $r$ planes in $R^{r+s}$. Imbed $G_{r, s}$ in a Euclidean space $R^{t}$ and let $U$ be a tubular neighborhood of $G_{r, s}$ with $\lambda$ the bundle over $U$ given by $\pi^{*} \gamma_{r, s}$, where $\pi: U \rightarrow G_{r, s}$ is the projection and $\gamma_{r, s}$ is the canonical bundle over $G_{r, s^{*}}$ Then the disc bundle $D\left(\lambda_{2}\right)$ is a manifold with boundary, and let $W$ be the double of $D(\lambda), f: X \rightarrow W$ being the composite of

$$
X \stackrel{g}{\longrightarrow} G_{r, s} \stackrel{i}{\longrightarrow} U \stackrel{0}{\longrightarrow} D(\lambda) \stackrel{j}{\longrightarrow} W
$$

with $i, j$ inclusions and 0 the zero section. Then $f^{*} \tau_{W}$ is stably equivalent to $\xi$, for $j^{*} \tau_{W} \cong \tau_{D(\lambda)}=\pi^{*} \lambda \oplus \pi^{*} \tau_{C^{i}}$ with $\pi: D(\lambda) \rightarrow U$ the projection, but $U$ is parallelizable so $\tau_{U}$ is trivial, so $f^{*} \tau_{W} \cong(i \circ g)^{*}(\lambda \oplus \operatorname{dim} U) \cong g^{*} \gamma_{r, 8} \oplus t$ which is by choice stably $\xi$. Letting $S$ be a disjoint union of spheres and $h: X \rightarrow S$ the union of point maps so that for each component $X_{0}$ of $X$ $h\left(X_{0}\right) \subset S^{q}$ with $r-q=\operatorname{dim} \xi \mid X_{0}$, one has $\phi([(W, f)],[(S, h)])=[\xi] \in K O(X)$. Thus $\phi$ is epic.

If $X$ is a closed manifold, $\phi$ is monic, for if $\alpha=([(M, f)],[(N, g)]) \in \mathfrak{B}(X)$ with $\phi(\alpha)=0$, then $f^{*} \tau_{M} \cong g^{*} \tau_{N}$ stably, with the manifolds having the same dimension (componentwise). Then $\alpha=\left(\left[\left(M \times X \times S^{r}, f \times 1 \times p t\right)\right]\right.$, $\left.\left[\left(N \times X \times S^{r}, g \times 1 \times p t\right)\right]\right), r>0$, and $X$ is imbedded in $M \times X \times S^{r}$ by $f \times 1 \times p t$ with normal bundle $f^{*} \tau_{M} \oplus r$ so $\left(M \times X \times S^{r}, f \times 1 \times p t\right)=$ $\left(S\left(f^{*} \tau_{M} \oplus(r+1)\right), \sigma\right)$ where $S$ is the sphere bundle and $\sigma$ is the section given by $x \rightarrow\left(0_{x}, 1\right) \in E\left(f^{*} \tau_{M I} \oplus r\right) \times R, E$ being the total space. Thus $\alpha=$ $\left(\left[\left(S\left(f^{*} \tau_{M} \oplus(r+1)\right), \sigma\right)\right],\left[\left(S\left(g^{*} \tau_{N^{\oplus}}(r+1)\right), \sigma\right)\right]\right)$ and for $r$ large $f^{*} \tau_{M} \oplus r \cong$ $g^{*} \tau_{N} \oplus r$, so $\alpha$ is the "zero" element in $\mathfrak{W}(X)$.

If $X$ is any finite complex, let $i: X \rightarrow R^{t}$ be an imbedding with $U \subset R^{t}$ a regular neighborhood collapsing onto $X$ by $c: U \rightarrow X$. $U$ is a compact manifold with boundary, and hence so is $V=\partial(U \times[0,1])$. One then has

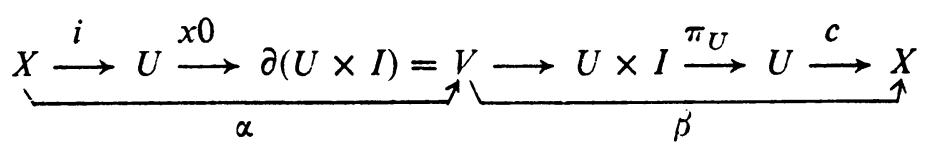


with $\beta \alpha=1$. One then has the diagram

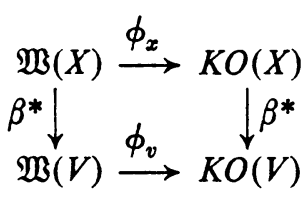

which commutes, with $\phi_{v}$ an isomorphism and both maps $\beta^{*}$ being monomorphisms onto direct summands. Thus $\phi_{x}$ is also monic.

This result provides a "cobordism theoretic" formulation of $K O$ theory. Using maps into stably almost complex manifolds in a similar fashion (with dimension given by dimension of the image manifold) one may see that $\mathfrak{W}^{c x}(X) \cong K(X)$. The points worth mentioning are that one uses the complex Grassmannian and that the manifold $V$ constructed in the last part of the proof is stably framed, hence stably almost complex.

Conner and Floyd [2] provides an entirely different relation between "cobordism" and $K$ theory, where "cobordism" is dual to stably almost complex bordism in the sense of spectrally defined homology theory.

It would be interesting to compute $(X) \mathfrak{N}_{*}$, but I have been unable to get anything nontrivial on it. The idea for this argument came from a remark of W. Browder, in his approach to bordism, that $B O$ is the universal manifold.

\section{REFERENCES}

1. M. F. Atiyah, Bordism and cobordism, Proc. Cambridge Philos. Soc. 57 (1961), 200-208. MR 23 \#A4150.

2. P. E. Conner and E. E. Floyd, The relation of cobordism to $K$-theories, Lecture Notes in Math., no. 28, Springer-Verlag, Berlin, 1966. MR 35 \#7344.

Department of Mathematics, University of Virginia, Charlottesville, Virginia 22903 